\title{
Chemical bonds from through-bridge orbital communications in prototype molecular systems
}

\author{
Roman F. Nalewajski
}

Received: 9 September 2010 / Accepted: 14 October 2010 / Published online: 30 October 2010 (C) The Author(s) 2010. This article is published with open access at Springerlink.com

\begin{abstract}
The indirect components of chemical interactions between atomic orbitals are explored within the Orbital Communication Theory of the chemical bond. The conditional probabilities for such through-bridge probability propagation and the associated entropy/information measures of the bond covalency are examined. The illustrative example of the bridge components of the chemical bonds between bridgehead carbons in small propellanes is discussed using the hybrid-orbital model. The bridge $\pi$-bonds in benzene and butadiene from the inter-orbital communications involving the single intermediate atomic orbitals are probed within the Hückel description and selected higher-orders of orbital bridges, involving several orbital intermediaries, are investigated.
\end{abstract}

Keywords Chemical bonds - Bonding mechanisms - Entropic bond indices . Information theory $\cdot$ Orbital bridges $\cdot P i$-electron systems · Propellanes · Through-bridge interactions · Through-space bonds · Wiberg bond-orders

\section{Introduction}

It has been recently argued [1-3] that the chemical interaction between two Atomsin-Molecules (AIM) has both the through-space and through-bridge components. The former reflects the direct interactions between such bonded atoms while the latter is realized indirectly, through the remaining atoms, which constitute a bridge for an

Throughout the paper $A$ denotes a scalar quantity, $\boldsymbol{A}$ stands for a row-vector, and $\mathbf{A}$ represents a square or rectangular matrix.

R. F. Nalewajski (凶)

Theoretical Chemistry Department, Jagiellonian University, R. Ingardena 3, 30-060 Cracow, Poland e-mail: nalewajs@chemia.uj.edu.pl 
effective chemical coupling between more distant AIM. The most efficient bridges for such an implicit bonding mechanism realized via atomic intermediaries are the real chemical bridges, originating from the basis functions contributed by the chemically bonded atoms connecting such "terminal" atoms in molecular system [3].

Thus, the bonded status of the given pair of atoms can be felt even at relatively large separations provided there exist real bridge(s) of direct chemical bonds connecting them. In other words, atoms exhibiting the vanishing direct chemical interaction can be still bonded indirectly, via AIM bridges. This novel mechanism has already been shown to have important implications for the bonding pattern of the $\pi$-interactions in hydrocarbons [3]. For example, in the $\pi$-system of benzene the ortho-carbons exhibit a strong Wiberg [4] bond-multiplicity measure of almost exclusively throughspace origin. The cross-ring interactions between the meta- and para-carbons where shown to be described by much smaller but practically equalized overall resultant bond-orders, being distinguished solely by the direct/indirect composition of these resultant multiplicities: the meta interactions are realized exclusively through bridges, while the para bonds exhibit strong direct and indirect components.

In the Orbital Communication Theory (OCT) [2,5-9] the "explicit" (through-space) bond component originates from the direct probability scattering between the interacting Atomic Orbitals (AO), measured by the corresponding conditional probability related to the square of the relevant element of the system density matrix, which couples the two basis functions, and hence to the associated Wiberg bond-order [4] contribution. In this information-theoretic (IT) [10-13] approach, which uses the standard communication-noise and information-flow descriptors [1,2] of the bond IT covalency and ionicity, respectively, the bonded atoms "communicate" between themselves in accordance with the electron delocalization pattern implied by the occupied (bonding) subspace of Molecular Orbitals (MO) resulting from the quantum-mechanical description of the system as a whole. In other words, this through-space bonding mechanisms involves a direct "conversation" between the two atoms in question. The "implicit" (through-bridges) bond component can be similarly viewed as resulting from the indirect information propagation via the bridge AIM. In a sense, while the through-space bonding channel reflects the direct "conversation" between AIM, the through-bridge channel(s) can be compared to a chatty talk reporting "hearsay", the "rumor" spread between the two atoms via the connecting chain of the AIM-intermediaries involved in the effective chemical bridge under consideration.

To summarize, one distinguishes in OCT the direct ("dialogue") and indirect ("gossip") origins of the chemical bond, which both contribute to the overall measure of the effective IT bond-order between the given pair of AIM. A similar description follows [1-3] from the Wiberg-type bond-multiplicities formulated in the MO theory [4,14-23]. In MO description the chemical interaction between, say, two (valence) $\mathrm{AO}$ or general basis functions originating from different atoms is strongly influenced by their direct overlap and interaction, which both condition the bonding effect experienced by electrons occupying their bonding combination in the molecule, compared to the non-bonding reference of electrons on separated AO. The "through-space" bonding mechanism is then associated with typical accumulation of valence electrons in the region between the two nuclei, called the bond-charge, due to the constructive interference between the two functions contributed by AIM. Indeed, such "shared" 
bond-charge is synonymous with the presence of the bond-covalency in this familiar (direct) chemical interaction.

This common possession of the spin-paired electrons by both atoms is also reflected by the familiar covalent VB structure. Similar effect of the bonding accumulation of the information densities relative to the promolecular distribution has been detected in maps of alternative measures of the information densities, e.g., of the entropy deficiency or the displacement in Shannon's entropy relative to the promolecular distribution [1-3,24-27]. In this description the complementary ionicity aspect is manifested by the MO polarization or-alternatively — by the participation of the orthogonal (independent) component of the ionic VB structure in the ground-state wave function.

The direct bonding interaction between neighboring atoms, reflected by the corresponding explicit bond-order of Wiberg, is thus generally associated with the presence of the bond-charge or the increase of information density between the two nuclei. However, for more distant atomic partners such an accumulation of valence electrons can be absent, e.g., in the cross-ring $\pi$-interactions in benzene or between the bridgehead carbon atoms in small propellanes, for which the "charge-shift" bonding mechanism [28], involving instantaneous charge fluctuations due to a strong resonance between covalent and ionic VB structures, has been proposed.

Alternatively, such bonding interaction lacking an accumulation of the bond-charge (information) can be also realized indirectly, through the neighboring AO intermediaries forming a "bridge" for an effective interaction between distant ("terminal") AO [3]. This indirect (through-bridge) mechanism reflects the implicit dependencies between AO resulting from their joint participation in the overall system of chemical bonds determined by the subspace of the occupied MO.

Thus, in the generalized outlook on the bond-order concept one identifies the chemical bond multiplicity as a measure of the statistical "dependence" (non-additivity) between orbitals on different atomic centers [1-3]. On one hand, this dependence between basis functions on different atoms is realized directly (through space), by the constructive interference of orbitals (probability amplitudes) on two atoms, which generally increases the electron density between them. On the other hand, it also has an indirect origin, through the dependence on orbitals of the remaining AIM used to construct the whole system of the occupied MO. Indeed, the mutually-bonding status of two basis functions can be felt even at large distances due to their involvement in chemical bonds with the chemically interacting AO intermediaries, which strongly participate in the localized bonds of the AIM bridge connecting the parent atoms, from which the two reference basis functions originate. These dependencies are due to the orthonormality relations involving the bonding subspace of the occupied MO, which determine the entire framework of chemical bonds in the molecule.

To summarize, each pair of AO or AIM exhibits the partial through-space and through-bridge components [3]. The bond-order of the former quickly vanishes with increasing inter-atomic separation, when the interacting $\mathrm{AO}$ are heavily engaged in forming chemical bonds with other atoms or remain non-bonding, thus describing the lone electron pairs. The latter can still assume appreciable values, when the remaining atoms form an effective bridge of the neighboring, chemically bonded atoms, which links the specified terminal AO/AIM in question [3]. Thus, the non-vanishing densitymatrix element coupling the two AO in the molecule, which in MO theory reflects 
their directly-bonding status, is not essential for the existence of their through-bridge interaction. The latter may exist even when the direct interaction vanishes, provided the two AO strongly couple to the chemically bonded chain of orbitals connecting them.

The previous analysis [3], using mainly the Wiberg [4] measure of bond multiplicities, has explicitly identified both these components in chemical interactions between $\mathrm{AO}$ by using the appropriate projections of basis functions onto the bonding subspace of MO, the scalar products of which determine in the SCF MO theory the associated elements of the system charge-and-bond-order (CBO) density matrix. It is the main purpose of the present analysis to develop the conditional probabilities between the specified pairs of AIM originating from communications through the remaining AO. The corresponding IT-covalencies originating from such indirect probability propagation in the molecule will be examined within OCT and illustrative applications to small propellanes and $\pi$-electron systems will be reported.

\section{Indirect conditional probabilities}

Let us reexamine probability scattering through AO bridges in the standard SCF MO theory. The network of chemical bonds is then determined by the occupied MO in the system ground-state. For simplicity we assume the closed-shell (cs) configuration of $N=2 n$ electrons in the standard spin-restricted Hartree-Fock (RHF) description, which involves $n$ lowest, doubly-occupied (orthonormal) MO. In the LCAO MO approach they are generated as linear combinations of the (Löwdin-orthogonalized) AO (basis functions) $\chi=\left(\chi_{1}, \chi_{2}, \ldots, \chi_{m}\right)=\left\{\chi_{i}\right\},\langle\chi \mid \chi\rangle=\left\{\delta_{i, j}\right\} \equiv \mathbf{I}$, contributed by the system constituent atoms:

$\boldsymbol{\varphi}=\left\{\varphi_{s}\right\}=\left[\left(\varphi_{1}, \varphi_{2}, \ldots, \varphi_{n}\right),\left(\varphi_{n+1}, \ldots \varphi_{m}\right)\right] \equiv\left(\boldsymbol{\varphi}^{o}, \boldsymbol{\varphi}^{v}\right)=\chi \mathbf{C}=\chi\left(\mathbf{C}^{o} \mid \mathbf{C}^{v}\right)$.

Here, the rectangular matrices $\mathbf{C}^{o}=\left\langle\chi \mid \varphi^{o}\right\rangle$ and $\mathbf{C}^{v}=\left\langle\chi \mid \varphi^{v}\right\rangle$ group the expansion (LCAO) coefficients of the $n$ occupied and $(m-n)$ virtual MO, respectively, to be determined from the iterative self-consistent-field (SCF) procedure. The full SCF LCAO MO matrix $\mathbf{C}$ is unitary, $\mathbf{C}^{\dagger}=\mathbf{C}^{-1}$, since it "rotates" orthonormal AO into the orthonormal $\mathrm{MO}$, and hence the inverse transformation reads: $\chi=\varphi \mathbf{C}^{\dagger}$. The basis set projections onto the bond-subspace $\varphi^{\circ}$,

$$
\left|\chi^{b}\right\rangle=\hat{\mathrm{P}}_{\varphi}^{o}|\chi\rangle=\left|\varphi^{o}\right\rangle\left\langle\varphi^{o} \mid \chi\right\rangle=\left|\varphi^{o}\right\rangle \mathbf{C}^{o \dagger}=\left\{\hat{\mathrm{P}}_{\varphi}^{o}|i\rangle=\left|i^{b}\right\rangle\right\}
$$

then determine the 1-density (CBO) matrix $\gamma$ :

$$
\begin{aligned}
\boldsymbol{\gamma} & =\mathbf{C d \mathbf { C } ^ { \dagger }}=2\left\langle\chi \mid \varphi^{o}\right\rangle\left\langle\varphi^{o} \mid \chi\right\rangle=2 \mathbf{C}^{o} \mathbf{C}^{o \dagger} \equiv 2\left\langle\chi\left|\hat{\mathrm{P}}_{\varphi}^{o}\right| \chi\right\rangle \\
& =2\left(\langle\chi| \hat{\mathrm{P}}_{\varphi}^{o}\right)\left(\hat{\mathrm{P}}_{\varphi}^{o}|\chi\rangle\right) \equiv 2\left\langle\chi^{b} \mid \chi^{b}\right\rangle
\end{aligned}
$$

where the diagonal matrix $\mathbf{d}=\left\{(2, s \leq n ; 0, s>n) \delta_{s, s^{\prime}}\right\}$ groups the MO occupations. The CBO matrix thus constitutes the AO representation of the projection operator $\hat{\mathrm{P}}_{\varphi}^{o}$ 
onto the bond-subspace $\varphi^{o}$. It thus satisfies the idempotency relation:

$$
(\gamma)^{2}=4\left\langle\chi\left|\hat{\mathrm{P}}_{\varphi}^{o}\right| \chi\right\rangle\left\langle\chi\left|\hat{\mathrm{P}}_{\varphi}^{o}\right| \chi\right\rangle=4\left\langle\chi\left|\left(\hat{\mathrm{P}}_{\varphi}^{o}\right)^{2}\right| \chi\right\rangle=4\left\langle\chi\left|\hat{\mathrm{P}}_{\varphi}^{o}\right| \chi\right\rangle=2 \gamma,
$$

since, in the molecular Hilbert space spanned by the basis set $\chi,|\chi\rangle\langle\chi| \hat{\mathrm{P}}_{\varphi}^{o}=\hat{\mathrm{P}}_{\varphi}^{o}$ or $|\chi\rangle\langle\chi|=1$.

The square of the off-diagonal CBO matrix element $\gamma_{i, j}$ linking two different AO $\chi_{i}$ and $\chi_{j}$, contributed by atoms $\mathrm{A}$ and $\mathrm{B}$, respectively, determines the contribution

$$
C \kappa_{i, j}=\gamma_{i, j} \gamma_{j, i}=4\left\langle j^{b} \mid i^{b}\right\rangle\left\langle i^{b} \mid j^{b}\right\rangle=4\left\langle j^{b}\left|\hat{\mathrm{P}}_{i}^{b}\right| j^{b}\right\rangle=4\left|\left\langle i^{b} \mid j^{b}\right\rangle\right|^{2} \equiv 4\left|S_{i, j}^{b}\right|^{2}
$$

to the overall Wiberg index [4] of the molecular bond-multiplicity between these atoms:

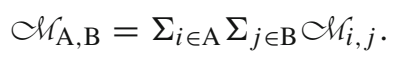

This quadratic bond-multiplicity concept has been subsequently extended [14-16] and generalized in terms of the bond-orders from the two-electron difference approach [17-23]. It follows from Eq. 5 that this "through-space" dependence between two AO located on different atoms originates from the direct "overlap" $S_{i, j}^{b}$ between the bond-projections $\left|i^{b}\right\rangle$ and $\left|j^{b}\right\rangle$ of the interacting orbitals,

$$
S_{i, j}^{b}=\left(\langle i| \hat{\mathrm{P}}_{\varphi}\right)\left(\hat{\mathrm{P}}_{\varphi}|j\rangle\right)=\left\langle i^{b} \mid j^{b}\right\rangle=\gamma_{i, j} / 2
$$

which reflect the overall involvement of these two basis functions in all chemical bonds in the molecular system under consideration.

The 1-matrix reflects the promoted, valence state of $\mathrm{AO}$ in the molecule, with the diagonal elements measuring the effective electron occupations of these basis functions, $\left\{N_{i}=\gamma_{i, i}=N p_{i}\right\}$, with probabilities $\boldsymbol{p}=\left\{p_{i}=\gamma_{i, i} / N\right\}$ of the basis functions occupancy in molecule: $\operatorname{Tr} \boldsymbol{\gamma}=N \Sigma_{i} p_{i}=N$. The off-diagonal CBO elements between $\mathrm{AO}$ on different atoms similarly reflect the bonding status of the direct interaction of the specified AO pair in the molecule, with the positive (negative) values signifying the bonding (anti-bonding) coupling between basis functions, and the vanishing bond-order $\gamma_{i, j}=0$ identifying their directly non-bonding chemical interaction, when $\left|i^{b}\right\rangle=0$ or $\left|j^{b}\right\rangle=0$. Thus, the "constructive" (bonding) interference between two AO, the basis functions of SCF MO calculations, requires the two AO in question to exhibit the positive product of their direct bond-projections, while the negative product value identifies their resultant "destructive" interference in the molecular bond system.

The 1-density matrix also determines the conditional probabilities for the direct information propagation in the AO information system, the key concept of OCT [2,5-9], in which the basis functions of SCF MO calculations provide a natural resolution level of the electron-assignment "events", appropriate for discussing the information scattering via the system chemical bonds. This AO communication network is 
then described by standard quantities developed in IT for real communication devices [10-13].

Due to the electron delocalization throughout the network of chemical bonds the transmission of "signals" about the electron-assignments to AO becomes randomly disturbed in the molecule, thus exhibiting typical communication "noise". Indeed, an electron initially attributed to the given AO in the channel "input" $\boldsymbol{a}=\left\{\chi_{i}\right\}$ can be later found with a non-zero probability at several locations in the molecular "output" $\boldsymbol{b}=\left\{\chi_{j}\right\}$. This feature of the electron delocalization is embodied in the conditional probabilities of the "outputs-given-inputs",

$$
\mathbf{P}(\boldsymbol{b} \mid \boldsymbol{a})=\left\{P(j \mid i)=P(i \wedge j) / p_{i}\right\}, \quad \Sigma_{j} P(j \mid i)=1 .
$$

The probabilities of simultaneously observing two AO in the system chemical bonds $\mathbf{P}(\boldsymbol{a} \wedge \boldsymbol{b})=\{P(i \wedge j)\}$ satisfy the following normalization:

$$
\Sigma_{i} P(i \wedge j)=p_{j}, \quad \Sigma_{j} P(i \wedge j)=p_{i}, \quad \Sigma_{i} \Sigma_{j} P(i \wedge j)=1
$$

where $p_{i}=\gamma_{i, i} / N$ stands for the AO probability in the molecule. These AO-pair probabilities are determined from the superposition-principle of quantum mechanics [29], supplemented by the "physical" projection onto the bond-subspace [2,6-9]:

$$
\mathbf{P}(\boldsymbol{b} \mid \boldsymbol{a})=\left\{P(j \mid i)=\left(2 \gamma_{i, i}\right)^{-1} \gamma_{i, j} \gamma_{j, i}=\left(2 \gamma_{i, i}\right)^{-1}\left(\gamma_{i, j}\right)^{2}\right\}
$$

Hence the associated joint-probability matrix:

$\mathbf{P}(\boldsymbol{a} \wedge \boldsymbol{b})=\left\{P(i \wedge j)=p_{i} P(j \mid i)=(2 N)^{-1} \gamma_{i, j} \gamma_{j, i}=(2 / N)\left\langle i\left|\hat{\mathrm{P}}_{\varphi}^{o}\right| j\right\rangle\left\langle j\left|\hat{\mathrm{P}}_{\varphi}^{o}\right| i\right\rangle\right\}$

The indirect probability scattering through the remaining basis functions $\chi^{\prime}=\left\{\chi_{k \neq(i, j)}\right\}$, which constitute the effective bridge for the specified AO pair $\chi_{i}$ (input) and $\chi_{j}$ (output) can be then determined as conditional probabilities of the underlying information cascade of Fig. 1, in which the input signal emitted at the input $a_{i}=\chi_{i}$ is propagated into the specified output $b_{j}=\chi_{j}$ through the admissible single-orbital bridges including all remaining AO: $\boldsymbol{c}(i, j)=\left\{c_{k}(i, j)=\chi_{k \neq(i, j)}\right\}$. The associated conditional probability for such a through-bridges propagation thus reads:

$$
\begin{aligned}
P[(j \mid i) \mid(c(i, j)] & =\sum_{k \neq(i, j)} P(k \mid i) P(j \mid k) \\
& =\sum_{l} P(l \mid i) P(j \mid l)-P(j \mid i)[P(i \mid i)+P(j \mid j)] \\
& =P^{2}(j \mid i)-\frac{1}{2} P(j \mid i)\left[\gamma_{i, i}+\gamma_{j, j}\right] \\
& \equiv P^{2}(j \mid i)-P(j \mid i) \gamma^{a v \cdot}(i, j),
\end{aligned}
$$


Fig. 1 The intermediate probability scattering between the specified AO events $a_{i}$ and $b_{j}$, in the channel input and output, respectively, through the AO intermediates $c(i, j)=\left\{c_{k}(i, j)\right\}$

Fig. 2 Effective sequential cascade of two information systems in AO resolution

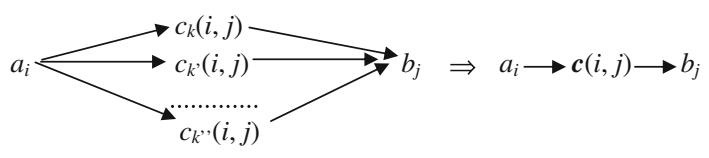

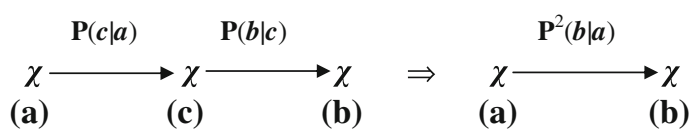

where the square of the $\mathrm{AO}$ conditional probabilities,

$$
\begin{aligned}
\mathbf{P}^{2}(\boldsymbol{b} \mid \boldsymbol{a}) & =\left\{P^{2}(j \mid i)=\left[\mathbf{P}^{2}(\chi \mid \chi)\right]_{i, j}=\mathbf{P}(\boldsymbol{c} \mid \boldsymbol{a}) \mathbf{P}(\boldsymbol{b} \mid \boldsymbol{c})\right]_{i, j}=\sum_{l} P(l \mid i) P(j \mid l) \\
& =\sum_{l} \frac{\gamma_{i, l}^{2} \gamma_{j, l}^{2}}{4 \gamma_{i, i} \gamma_{l, l}}
\end{aligned}
$$

characterizes the complete sequential AO-cascade (Fig. 2) involving all orbital intermediaries [30]. It follows from Eq. 12 that the intermediate probability propagation via the full "bridge" consisting off all the remaining $\mathrm{AO}, \chi_{i} \rightarrow \boldsymbol{c}(i, j) \rightarrow \chi_{j}$, is determined by the corresponding squared-matrix probability $P^{2}(j \mid i)$ of the sequential AO cascade corrected by the product of the direct-scattering probability $P(j \mid i)$ and the average occupation $\gamma^{a v} \cdot(i, j)$ of the specified input and output AO in the molecule.

Of interest also are the dominating partial bridges consisting of $\mathrm{AO}$ contributed by the sequence of the chemically bonded AIM connecting the specified two atoms in the molecular input and output, respectively. Such probabilities, be it for the different normalization convention, have been preliminarily examined in the previous analysis [3], using the appropriate sequence of the $\mathrm{AO}$ projections.

It follows from Eq. 9 that for the single $\mathrm{AO} \chi_{k}$ in the bridge the present sequential approach gives:

$$
P[(j \mid i) \mid k]=P(k \mid i) P(j \mid k)=\frac{\gamma_{i, k}^{2} \gamma_{j, k}^{2}}{4 \gamma_{i, i} \gamma_{k, k}}, \quad \Sigma_{j} P[(j \mid i) \mid k]=P(k \mid i) .
$$

For the parallel two AO intermediaries of Fig. 3a one similarly finds:

$P[(j \mid i) \mid(k, l)]=P(k \mid i) P(j \mid k)+P(l \mid i) P(j \mid l)=\frac{1}{4 \gamma_{i, i}}\left(\frac{\gamma_{i, k}^{2} \gamma_{j, k}^{2}}{\gamma_{k, k}}+\frac{\gamma_{i, l}^{2} \gamma_{j, l}^{2}}{\gamma_{l, l}}\right)$, 
Fig. 3 The intermediate probability scattering from $\chi_{i}$ to $\chi_{j}$ via the two-AO bridges $\left\{\chi_{k}, \chi_{l}\right\}$ : parallel (Panel a) and sequential (Panel b) (a)

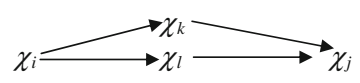

(b)

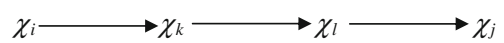

while the sequential two-AO bridge of Fig. 3b gives:

$$
P[(j \mid i) \mid k \rightarrow l]=P(k \mid i) P(l \mid k) P(j \mid l)=\frac{\gamma_{i, k}^{2} \gamma_{l, k}^{2} \gamma_{j, l}^{2}}{8 \gamma_{i, i} \gamma_{k, k} \gamma_{l, l}} .
$$

\section{Orbital model of the central bond in small propellanes}

Let us examine the patterns of chemical bonds in the representative [1.1.1] and [2.2.2] propellanes (Fig. 4), incuding a single and double carbon bridges, respectively. In the minimum basis set the bond structure in these two molecular systems can be understood in terms of the localized MO resulting from interactions between directed (hybrid) orbitals on neighboring atoms and the non-bonding electrons occupying such hybrid AO. In the smallest [1.1.1] system the nearly tetrahedral $\left(h=s p^{3}\right)$ hybridization on both bridgehead and bridging carbons is required to form chemical bonds of the three carbon bridges and to accommodate two hydrogens on each bridge-carbons. Thus three $s p^{3}$ hybrids on each of the bridgehead atoms are used to form the chemical bonds with the bridge carbons and the fourth hybrid is directed away from the central-bond region, between the two bridgehead carbons, thus remaining non-bonding and singly-occupied. In the [2.2.2] propellane the two central carbons acquire a nearly trigonal $\left(h^{\prime}=s p^{2}\right)$ hybridization, to form bonds with the bridge neighbours, each with a single $2 p_{\sigma}$ orbital directed along the central-bond axis, which has not been used in this hybridization scheme, now being available to form a strong through-space component of the overall multiplicity of the $\mathrm{C}^{\prime}-\mathrm{C}^{\prime}$ bond. This explains the missing through-space component in the smaller (diradical) propellane and its presence in the larger system [1-3,24]. The same conclusion follows from the information-probes of the direct bonding pattern in these molecules [1,2,24,27].

(a)

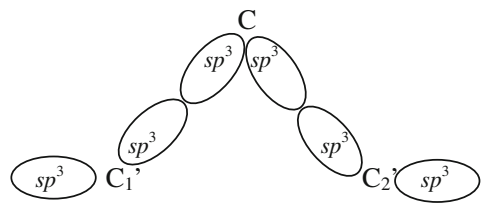

(b)

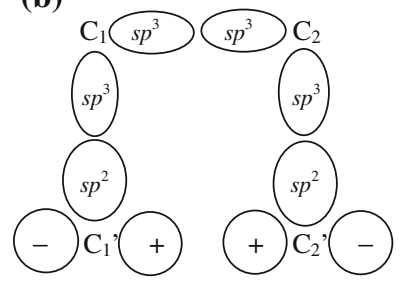

Fig. 4 Schematic diagrams rationalizing the patterns of the localized bonds in [1.1.1] (Panel a) and [2.2.2] (Panel b) propellanes; the bridgehead carbon atoms are primed 
In this qualitative picture each directed AO participates in a single localized, twocentre (doubly-occupied) bonding MO, which allows one to estimate the diatomic CBO matrix elements determining the direct and indirect components of the central bonds in these two propellanes:

$$
\begin{aligned}
& \gamma_{p, p}=\left(1+S_{p, p}^{\sigma}\right)^{-1} \approx 0.78, \quad \gamma_{h, h}=\left(1+S_{h, h}\right)^{-1} \approx 0.60, \\
& \gamma_{h, h^{\prime}}=\left(1+S_{h, h^{\prime}}\right)^{-1} \approx 0.60,
\end{aligned}
$$

where $S_{p, p}^{\sigma}, S_{h, h}$ and $S_{h, h^{\prime}}$ stand for the overlap integrals between two $2 p_{\sigma}$ orbitals and between the indicated hybrid-AO, respectively. In the preceding equation these orbital overlaps have been realistically estimated using the standard overlap integrals between valence orbitals on carbon atoms in ethane (single $\mathrm{C}-\mathrm{C}$ bond): $S_{s, s}=0.36, S_{s, p}^{\sigma}=0.42, S_{s, p}^{\sigma}=0.42, S_{p, p}^{\sigma}=0.28$, giving rise to the associated standard overlaps between hybrid AO : $S_{h, h}^{\sigma}=0.66, S_{h, h^{\prime}}^{\sigma}=0.67$.

Hence, the direct Wiberg component of the central bond in the [2.2.2] system,

$$
C S_{1^{\prime}, 2^{\prime}} \approx\left(1+S_{p, p}^{\sigma}\right)^{-2}=0.62
$$

and the indirect contribution due to three (double-carbon) bridges [3],

$$
\alpha \kappa_{1^{\prime}, 2^{\prime}}(\text { bridges })=3 \propto \kappa_{\mathrm{C}_{1}^{\prime}, \mathrm{C}_{1}} \propto \kappa_{\mathrm{C}_{1}, \mathrm{C}_{2}} \propto \kappa_{\mathrm{C}_{2}, \mathrm{C}_{2}^{\prime}} \approx 3\left(1+S_{h, h^{\prime}}^{\sigma}\right)^{-4}\left(1+S_{h, h}^{\sigma}\right)^{-2}=0.14,
$$

which give rise to the total bond multiplicity:

$$
d\left(1^{\prime}-2^{\prime}\right)=d r_{1^{\prime}, 2^{\prime}}+d r_{1^{\prime}, 2^{\prime}}(\text { bridges }) \approx 0.76 \text {. }
$$

The corresponding indirect (total) component for the [1.1.1] system reads:

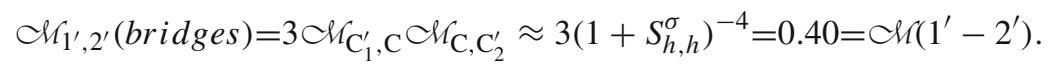

Therefore, the smaller system is predicted to exhibit higher through-bridge component, compared to larger propellane, with the latter generating greater overall bond-order. This trend is also reflected by numerical SCF and DFT calculations [1-3,24].

Finally, let us examine the associated conditional entropy (communication noise) contributions, reflecting the associated IT bond-orders due to through-bridge covalencies. The conditional probabilities of Eqs. 14 and 16, due to a single bridge in the [1.1.1] and [2.2.2] propellanes, respectively, read:

$$
\begin{aligned}
P\left[\left(\mathrm{C}_{2}^{\prime} \mid \mathrm{C}_{1}{ }^{\prime}\right) \mid \mathrm{C}\right] & \approx \frac{1}{4}\left(\gamma_{h, h}\right)^{4}=0.0333 \quad \text { and } \\
P\left[\left(\mathrm{C}_{2}^{\prime} \mid \mathrm{C}_{1}^{\prime}\right) \mid \mathrm{C}_{1} \rightarrow \mathrm{C}_{2}\right] & \approx \frac{1}{8}\left(\gamma_{h, h}\right)^{2}\left(\gamma_{h, h^{\prime}}\right)^{4}=0.0058 .
\end{aligned}
$$

They again reflect a higher through-bridge propagation of electron probability in the single carbon bridge. These probabilities generate the associated entropies due to three 
identical (parallel) bridges, which measure the bridge IT-covalencies (in bits) of the central bond in these two molecular systems:

$$
\begin{aligned}
& \mathrm{S}_{1^{\prime}, 2^{\prime}}(\text { bridges }) \approx 3\left(-0.0333 \log _{2} 0.0333\right)=0.49 \quad \text { and } \\
& \mathrm{S}_{1^{\prime}, 2^{\prime}}(\text { bridges }) \approx 3\left(-0.0058 \log _{2} 0.0058\right)=0.13,
\end{aligned}
$$

which compare favorably with the corresponding Wiberg estimates of Eqs. 21 and 19, respectively.

We thus conclude that the entropic and Wiberg measures of the through-bridge component of the central bond covalency in this simple model of the electronic structure in the representative [1.1.1] and [2.2.2] propellanes are in general agreement with one another thus providing consistent insights into the novel through-bridge bond components in these prototype molecular systems.

\section{Communications in $\pi$-electron systems through single-AO bridges}

Next, let us reexamine the indirect $\pi$-bonds between carbon atoms in benzene and butadiene, using the occupied MO from the familiar Hückel theory. We begin with a brief summary of the main predictions from the previous analysis [3] of the Wiberg bond-orders in these molecules.

The density matrix in benzene is summarized by the following elements of the CBO matrix:

$$
\gamma_{i, i}=1, \quad \gamma_{i, i+1}=2 / 3, \quad \gamma_{i, i+2}=0, \quad \gamma_{i, i+3}=-1 / 3 .
$$

This density matrix generates the following through-space $\pi$ bond-orders of Wiberg:

$$
d r_{i, i+1}=0.44, \quad c K_{i, i+2}=0, \quad c K_{i, i+3}=0.11,
$$

predicting the vanishing direct bond-multiplicities between the two meta-carbons. In the previous study [3] we have demonstrated that these explicit $\pi$-bonds are supplemented by the indirect interactions via the remaining carbon atoms in the benzene ring:

$d \kappa_{i, i+1}($ bridges $)=0.06, \quad C \kappa_{i, i+2}($ bridges $) \cong 0.30, \quad \alpha \kappa_{i, i+3}($ bridges $) \cong 0.18$,

thus predicting the associated total measures of the chemical $\pi$ interactions in benzene:

$$
\operatorname{cr}(\text { para }) \cong \operatorname{Cr}(\text { meta })=0.3<\operatorname{Cr}(\text { ortho })=0.5 \text {. }
$$

One observes the differences in their compositions: the para interactions exhibit comparable through-space and through-bridge components, the meta multiplicities are realized through bridges only, while the strongest ortho bond-orders have practically direct, through-space origin. 
For the consecutive numbering of carbon atoms in butadiene the off-diagonal part of the CBO matrix in Hückel approximation is fully characterized by the following elements:

$$
\gamma_{1,2}=\gamma_{3,4}=2 / \sqrt{5}, \quad \gamma_{1,3}=\gamma_{2,4}=0, \quad \gamma_{1,4}=-1 / \sqrt{5}, \quad \gamma_{2,3}=1 / \sqrt{5},
$$

which determine the associated through-space bond-orders of Wiberg:

$$
\alpha r_{1,2}=\alpha r_{3,4}=0.80, \quad c r_{1,3}=\alpha r_{2,4}=0, \quad \alpha r_{1,4}=\alpha r_{2,3}=0.20 .
$$

Again, this artificial distinction of the (1-3) and (2-4) interactions as $\pi$ non-bonding is remedied by the inclusion of the indirect bond components:

$d \kappa_{1,2}($ bridges $)=0.03, \quad \mathrm{Cr}_{1,3}($ bridges $)=0.32, \quad \mathrm{~d} \kappa_{1,4}($ bridges $)=0.13$,

which generate the following resultant bond orders:

$$
\begin{aligned}
& \operatorname{ch}(1-2)=\operatorname{ch}(3-4)=0.83, \quad \operatorname{ch}(1-3)=\operatorname{ch}(2-4)=0.32, \\
& \operatorname{ch}(1,4)=\operatorname{ch}(2,3)=0.33 .
\end{aligned}
$$

The strongest, terminal bonds (1-2) and (3-4) are almost exclusively of the throughspace origin, the $\pi$-bonds (1-3) and (2-4) connecting the second-neighbors exhibit the pure through-bridge character, while the remaining bonds (1-4) and (2-3) include comparable direct and indirect components.

Turning now to the orbital communications we recall, that the symmetry-unrelated, direct conditional probabilities [Eq. 10] in benzene,

$$
\begin{aligned}
P(i \mid i) & =1 / 2, & P(i+1 \mid i)=2 / 9, \\
P(i+2 \mid i) & =0, & P(i+3 \mid i)=1 / 18,
\end{aligned}
$$

define the through-space AO-communications for this molecule. The non-vanishing probabilities for butadiene read:

$$
\begin{array}{ll}
P(i \mid i)=1 / 2, & P(2 \mid 1)=P(4 \mid 3)=2 / 5 \\
P(3 \mid 1)=P(4 \mid 2)=0, & P(4 \mid 1)=P(3 \mid 2)=1 / 10 .
\end{array}
$$

The corresponding elements of the squared conditional probability matrices [Eq. 13], characterizing the sequential cascade of Fig. 2, for benzene,

$$
\begin{aligned}
P^{2}(i \mid i) & =19 / 54, & P^{2}(i+1 \mid i)=2 / 9, \\
P^{2}(i+2 \mid i) & =2 / 27, & P^{2}(i+3 \mid i)=1 / 18,
\end{aligned}
$$


Fig. 5 Indirect communications between terminal (identified by asterisk) ortho (Panel a), meta (Panel b) and para (Panel c) carbons in benzene, through the single $\pi$-AO intermediate (a)

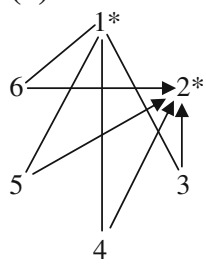

(b)

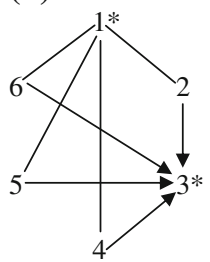

(c)

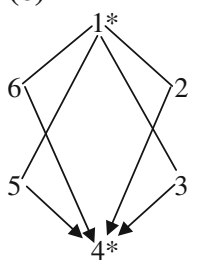

and butadiene,

$$
\begin{array}{ll}
P^{2}(i \mid i)=21 / 50, & P^{2}(2 \mid 1)=P^{2}(4 \mid 3)=2 / 5, \\
P^{2}(3 \mid 1)=P^{2}(4 \mid 2)=2 / 25, & P^{2}(4 \mid 1)=P^{2}(3 \mid 2)=1 / 10,
\end{array}
$$

then determine the indirect probability scatterings between these orbitals through all admissible single $\mathrm{AO}$ bridges including the remaining AO.

These additional degrees-of-freedom for communications between different $\pi$-AO, through the single member of the $\mathrm{AO}$ subset $\boldsymbol{c}(i, j)$ combining all remaining basis functions, are then given by the associated probabilities of Eq. 12. All these intermediate $\pi$-AO communications in benzene are illustrated in Fig. 5.

For benzene one finds

$$
\begin{aligned}
P[(i+1 \mid i) \mid \boldsymbol{c}(i, i+1) & =P[(i+3 \mid i) \mid \boldsymbol{c}(i, i+3)]=0, \\
P[(i+2 \mid i) \mid \boldsymbol{c}(i, i+2)] & =2 / 27,
\end{aligned}
$$

while these implicit communications in butadiene read:

$$
\begin{aligned}
& P[(2 \mid 1) \mid(3,4)]=P[(4 \mid 3) \mid(1,2)]=P[(4 \mid 1) \mid(2,3)]=P[3 \mid 2) \mid(1,4)]=0, \\
& P[(3 \mid 1) \mid(2,4)]=P[(4 \mid 2) \mid(1,3)]=2 / 25,
\end{aligned}
$$

Therefore, these results for the complete bridge of sequential probability propagation between two $\pi$-AO through the single orbital of the set combining the remaining basis functions predict vanishing single-AO bridge communications between the two orthoand para-carbons, with only meta-carbons exhibiting a non-vanishing indirect probability scattering. A similar trend is observed for butadiene, with only the $1 \rightarrow 3$ and $2 \rightarrow 4$ communications, which exhibit the vanishing direct component, now acquiring the indirect communication links.

The additional, indirect IT-covalency between two meta carbons in benzene is thus reflected by the associated conditional entropy (noise) descriptor:

$$
\mathrm{S}_{i, i+2}[c(i, i+2)]=-(2 / 27) \log _{2}(2 / 27)=0.28 \text { bits. }
$$

The indirect entropic covalency of the 1-3 and 2-4 IT bond-orders in butadiene similarly reads:

$$
\mathrm{S}_{1,3}(2,4)=\mathrm{S}_{2,4}(1,3)=-(2 / 25) \log _{2}(2 / 25)=0.29 \text { bits. }
$$


These implicit (total) bond IT-covalencies can be compared with the direct (total) entropies of the remaining two-orbital interactions in benzene,

$$
\begin{aligned}
& \mathrm{S}_{i, i+1}=-(2 / 9) \log _{2}(2 / 9)=0.48 \text { bits } \\
& \mathrm{S}_{i, i+3}=-(1 / 18) \log _{2}(1 / 18)=0.23 \text { bits }
\end{aligned}
$$

and in butadiene:

$$
\begin{aligned}
& S_{1,2}=S_{3,4}=-(2 / 5) \log _{2}(2 / 5)=0.53 \text { bits } \\
& S_{1,4}=S_{2,3}=-(1 / 10) \log _{2}(1 / 10)=0.33 \text { bits. }
\end{aligned}
$$

Therefore, the present perspective, combining the entropy-covalencies due to the direct $\mathrm{AO}$ communications and indirect probability propagations via single-AO bridges of all remaining basis functions, gives even more dichotomous distinction of diatomic $\pi$-interactions in these two molecules, compared to that resulting from all admissible bridges in the AO information system [3]. The bridge contributions now correct only the atomic pairs, which do not interact directly: in benzene $S_{i, i+2}[c(i, i+2)] \cong 0.3$ bits and in butadiene $S_{1,3}(2,4)=S_{2,4}[1,3] \cong 0.3$ bits. One again observes, that this indirect correction is of the order of the weaker direct bonds in these molecules.

\section{Higher orders of bridge communications in benzene}

It follows from Eq. 36 that the effective probabilities $\mathbf{Q}^{S}=\left\{Q_{i \rightarrow j}^{S}\{k \neq(i, j)\}\right.$ of the information scattering between the specified pair of the input $(i)$ and output $(j)$ $\mathrm{AO}$, through the single $(S)$-AO bridges including all the remaining basis functions $\left\{k_{\neq}(i, j)\right\}$ (see Fig. 6a) read:

(a)

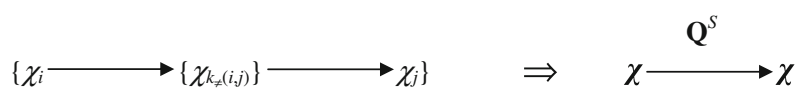

(b)

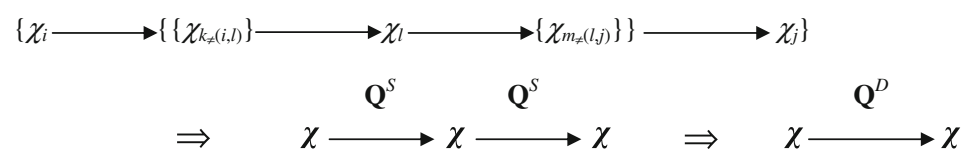

(c) $\left\{\chi_{i}\right.$

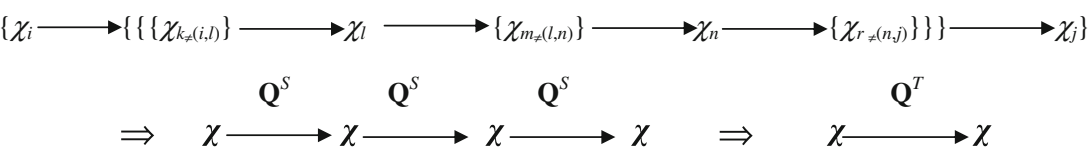

Fig. 6 The intermediate probability scattering $\chi_{i} \rightarrow \chi_{j}$ via the AO bridges including all single $(S)\left\{\chi_{k}\right\}$ (Panel a), double $(D)\left\{\chi_{k} \rightarrow \chi_{m}\right\}$ (Panel b), and triple $(T)\left\{\chi_{k} \rightarrow \chi_{m} \rightarrow \chi_{r}\right\}$ (Panel c) AO in the benzene ring, generating the associated conditional-probability matrices $\mathbf{Q}^{S}, \mathbf{Q}^{D}, \mathbf{Q}^{T}$, respectively 


$$
\mathbf{Q}^{S}=\left[\begin{array}{llllll}
0 & 0 & q & 0 & q & 0 \\
0 & 0 & 0 & q & 0 & q \\
q & 0 & 0 & 0 & q & 0 \\
0 & q & 0 & 0 & 0 & q \\
q & 0 & q & 0 & 0 & 0 \\
0 & q & 0 & q & 0 & 0
\end{array}\right], \quad q=2 / 27
$$

One can also envisage higher orders of AO bridges in which the specified $i \rightarrow j$ probability is propagated via the consecutive scatterings involving two (Fig. 6b) or three (Fig. 6c) AO bridges in the benzene ring. The associated conditional probability matrices are then determined by the corresponding powers of $\mathbf{Q}^{S}$ (see Fig. 6b,c):

$$
\begin{aligned}
& \mathbf{Q}^{D}=\left(\mathbf{Q}^{S}\right)^{2}=\left\{Q_{i \rightarrow j}^{D}[\{k \neq(i, l)\} \rightarrow\{m \neq(l, j)\}]\right. \\
& \left.=\sum_{l} Q_{i \rightarrow l}^{S}\{k \neq(i, l)\} Q_{l \rightarrow j}^{S}\{m \neq(l, j)\}\right\} \\
& =\left[\begin{array}{cccccc}
2 q^{2} & 0 & q^{2} & 0 & q^{2} & 0 \\
0 & 2 q^{2} & 0 & q^{2} & 0 & q^{2} \\
q^{2} & 0 & 2 q^{2} & 0 & q^{2} & 0 \\
0 & q^{2} & 0 & 2 q^{2} & 0 & q^{2} \\
q^{2} & 0 & q^{2} & 0 & 2 q^{2} & 0 \\
0 & q^{2} & 0 & q^{2} & 0 & 2 q^{2}
\end{array}\right], \\
& \mathbf{Q}^{T}=\left(\mathbf{Q}^{S}\right)^{3}=\left\{Q_{i \rightarrow j}^{T}[\{k \neq(i, l)\} \rightarrow\{m \neq(l, n)\} \rightarrow\{r \neq(n, j)\}]\right. \\
& =\sum_{l} \sum_{n} Q_{i \rightarrow l}^{S}\left\{k \neq(i, l) Q_{l \rightarrow n}^{S}\{m \neq(l, n)\} Q_{n \rightarrow j}^{S}\{r \neq(n, j)\}\right\} \\
& =\left[\begin{array}{cccccc}
2 q^{3} & 0 & 3 q^{3} & 0 & 3 q^{3} & 0 \\
0 & 2 q^{3} & 0 & 3 q^{3} & 0 & 3 q^{3} \\
3 q^{3} & 0 & 2 q^{3} & 0 & 3 q^{3} & 0 \\
0 & 3 q^{3} & 0 & 2 q^{3} & 0 & 3 q^{3} \\
3 q^{3} & 0 & 3 q^{3} & 0 & 2 q^{3} & 0 \\
0 & 3 q^{3} & 0 & 3 q^{3} & 0 & 2 q^{3}
\end{array}\right],
\end{aligned}
$$

These expressions demonstrate that these indirect communications fast decay with the bridge order $O=S, D, T, \ldots \equiv 1,2,3, \ldots$, with the non-vanishing terms of $\mathbf{Q}^{O}$ being determined by contributions of the order $\mathrm{O}\left(q^{O}\right)$. This enhances the importance of conclusions in Sect. 4, based upon the single bridges $O=S$, which determine the largest contributions to indirect communications between $\mathrm{AO}$.

\section{Conclusion}

In this work we have further explored the through-bridge mechanism of bonding interactions in molecular systems, which has been first conjectured to explain the numerical bond orders for propellane systems [1-3]. The present IT analysis and 
previous Wiberg-type treatment [3] of both the explicit and implicit chemical interactions in molecules confirm the presence of both these bond components. The former, more familiar bonding mechanism is associated with an accumulation of the electronic charge between bonded atoms. As conditioned by the direct overlap between the interacting orbitals it is possible only at relatively short distances between AIM. The latter does not require the presence of such a bond-charge and depends on the existence of the real bridge of chemically bonded atoms between the interacting AIM. As such it can be effected at larger separations between atoms, which may have important implications for biological and solid-state systems.

The bottom-line of both these studies is that the chemical bonding between two AO can in fact be realized despite the vanishing CBO matrix element coupling directly these basis functions in the molecule, provided that they both exhibit the non-vanishing density matrix elements with the bridge basis functions. In other words, the two AO may exhibit the indirect chemical bonding when they strongly couple to other directly bonded basis functions.

The simple orbital model of such direct and indirect interactions in small propellanes further confirms the apparent existence of the trough-bridge bond even in the smallest [1.1.1] system lacking the direct bond component, thus offering an explanation of the experimentally conjectured central bonding in this molecule despite the absence of the charge/information accumulation between central (bridgehead) carbons. This constitutes an additional insight into the bond pattern in these molecules, alternative to the VB-inspired charge-shift mechanism [28] of the instantaneous charge fluctuations between the central carbon atoms, invoked to explain the existence of "some" chemical bonding between the central carbons in the smallest propellane.

Both the Wiberg-type bond-order description and OCT treatment of such indirect bonding mechanism through the orbital/AIM intermediaries called "bridges" has been shown to also rationalize the $\pi$-bonding patterns in benzene and butadiene, by removing some artifacts of the traditional (direct) bond-order description, e.g., of the cross-ring interactions in benzene. The overall interactions of the two meta-carbons, lacking the direct (through-space) component, have been shown to amount to about 0.3 bond-order, thus being approximately of the same magnitude as the resultant bond multiplicity predicted for the two carbons in the mutual para-positions. The same extra IT-covalency follows from the OCT using the conditional-probability corrections due to bridging orbitals in the carbon ring. The dominant ortho-interactions in benzene and terminal $\pi$ bonds in butadiene have been shown to be almost exclusively of the through-space character, while the second-neighbor interactions in butadiene and meta interactions in benzene were found to be of the pure through-bridges origin.

The novel, indirect mechanism adds to the diversity and complexity of the chemical interactions in molecular systems, and offers an alternative perspective on some controversial chemical bonds in molecules, e.g., the central bond problem in propellanes. We recall that both the Shannon-type information densities $[1,2,24]$ and the contragradience criterion [2,31-35], related to the Electron Localization Function [27,36-38], fail to detect the presence of the direct chemical bond in the smallest propellane. The indirect bond concept has also been shown to remove some artifacts of the over-simplified approach to conjugated $\pi$ bonds based solely upon the through-space mechanism. 
Open Access This article is distributed under the terms of the Creative Commons Attribution Noncommercial License which permits any noncommercial use, distribution, and reproduction in any medium, provided the original author(s) and source are credited.

\section{References}

1. R.F. Nalewajski, Information Theory of Molecular Systems (Elsevier, Amsterdam, 2006), and refs. therein

2. R.F. Nalewajski, Information Origins of the Chemical Bond (Nova, New York, 2010), and refs. therein

3. R.F. Nalewajski, Through-space and through-bridge components of chemical bonds. J. Math. Chem. doi:10.1007/s10910-010-9747-6

4. K.A. Wiberg, Tetrahedron 24, 1083 (1968)

5. R.F. Nalewajski, J. Phys. Chem. A 104, 11940

6. R.F. Nalewajski, D. Szczepanik, J. Mrozek, Adv. Quant. Chem. (in press)

7. R.F. Nalewajski, Int. J. Quantum Chem. 109, 425, 2495 (2009)

8. R.F. Nalewajski, J. Math. Chem. 47, 692, 808 (2010)

9. R.F. Nalewajski, Use of the bond-projected superposition principle in determining the conditional probabilities of orbital events in molecular fragments, J. Math. Chem. doi:10.1007/s10910-010-9766-3

10. C.E. Shannon, Bell Syst. Tech. J. 27, 379, 623 (1948)

11. C.E. Shannon, W. Weaver, The Mathematical Theory of Communication (University of Illinois, Urbana, 1949)

12. N. Abramson, Information Theory and Coding (McGraw-Hill, New York, 1963)

13. P.E. Pfeifer, Concepts of Probability Theory, 2nd edn. (Dover, New York, 1978)

14. M.S. Gopinathan, K. Jug, Theor. Chim. Acta (Berl.) 63, 497, 511 (1983)

15. K. Jug, M.S. Gopinathan, ed. by Z.B. Maksić Theoretical Models of Chemical Bonding, Vol. II (Springer, Heidelberg, 1990), p. 77

16. I. Mayer, Chem. Phys. Lett. 97, 270 (1983)

17. R.F. Nalewajski, A.M. Köster, K. Jug, Theoret. Chim. Acta (Berl.) 85, 463 (1993)

18. R.F. Nalewajski, J. Mrozek, Int. J. Quantum Chem. 51, 187 (1994)

19. R.F. Nalewajski, S.J. Formosinho, A.J.C. Varandas, J. Mrozek, Int. J. Quantum Chem. 52, 1153 (1994)

20. R.F. Nalewajski, J. Mrozek, G. Mazur, Can. J. Chem. 100, 1121 (1996)

21. R.F. Nalewajski, J. Mrozek, A. Michalak, Int. J. Quantum Chem. 61, 589 (1997)

22. J. Mrozek, R.F. Nalewajski, A. Michalak, Polish J. Chem. 72, 1779 (1998)

23. R.F. Nalewajski, Chem. Phys. Lett. 386, 265 (2004)

24. R.F. Nalewajski, E. Broniatowska, J. Phys. Chem. A. 107, 6270 (2003)

25. R.F. Nalewajski, E. Świtka, A. Michalak, Int. J. Quantum. Chem. 87, 198 (2002)

26. R.F. Nalewajski, E. Świtka, Phys. Chem. Chem. Phys. 4, 4952 (2002)

27. R.F. Nalewajski, A.M. Köster, S. Escalante, J. Phys. Chem. A 109, 10038 (2005)

28. S. Shaik, D. Danovich, W. Wu, P.C. Hiberty, Nature Chem. 1, 443 (2009)

29. P.A.M. Dirac, The Principles of Quantum Mechanics, 4th edn. (Clarendon, Oxford, 1958)

30. R.F. Nalewajski, J. Math. Chem. 45, 607 (2009)

31. R.F. Nalewajski, Int. J. Quantum Chem. 108, 2230 (2008)

32. R.F. Nalewajski, P. de Silva, J. Mrozek, in Kinetic Energy Functional, ed. by A. Wang, T. Wesołowski (World Scientific, Singapore, 2009), in press

33. R.F. Nalewajski, J. Math. Chem. 47, 667 (2010)

34. R.F. Nalewajski, P. de Silva, J. Mrozek, J. Mol. Struct.: THEOCHEM 954, 57 (2010)

35. R.F. Nalewajski, J. Math. Chem. 47, 709 (2010)

36. A.D. Becke, K.E. Edgecombe, J. Chem. Phys. 92, 5397 (1990)

37. B. Silvi, A. Savin, Nature 371, 683 (1994)

38. A. Savin, R. Nesper, S. Wengert, T.F. Fässler, Angew. Chem. Int. Ed. Engl. 36, 1808 (1997) 